Palabra Clave (La Plata), octubre 2017, vol. 7, n 1, e037. ISSN 1853-9912

Universidad Nacional de La Plata.

Facultad de Humanidades y Ciencias de la Educación.

Departamento de Bibliotecología

\title{
Gestión de la información en las revistas de Brasil y Argentina: un análisis de la última década
}

\author{
Information management in the Brazil and Argentina \\ journals: an analysis of the last decade
}

\section{João Arlindo dos Santos Neto *, Juliana Cardoso dos Santos *, Natalia Marinho do Nascimento *}

* Universidade Estadual Paulista, Brasil | santosneto@uel.br, julimath21@gmail.com, nmarinhon@gmail.com

\section{PALABRAS CLAVE}

Gestión de la información

Artículos de revistas

Modelo de gestión de la Información

Estudio bibliométrico

Brasil

Argentina

\section{KEYWORDS}

Information management

Scientific articles

Models of information management

Bibliometric study

Brazil

Argentina

\begin{abstract}
RESUMEN
Percibir cómo la gestión de la información está siendo trabajada por las revistas del área auxilia tanto en el crecimiento de la propia área, así como en la de los profesionales que en ella actúan. El objetivo general de esta comunicación fue realizar un análisis minucioso de los artículos de las revistas con estrato Qualis Capes A1, A2 y B1 del área de Comunicación e información, direccionados para la temática de la Gestión de la Información, de Brasil y de Argentina. Con relación a los objetivos específicos, se buscó identificar cuáles son las revistas que más publicaron artículos en la temática; descubrir en qué año hubo un mayor número de publicaciones; conocer quiénes son los autores más productivos; encasillar las palabras-clave más empleadas en los artículos; averiguar los procedimientos metodológicos utilizados y la manera como los textos recuperados están discutiendo la temática gestión de la información, así como sus modelos en estos contextos. En cuanto a la metodología, el artículo es de naturaleza exploratoria con abordaje tanto cualitativa como cuantitativa, a partir de una revisión bibliográfica sobre los temas información y competitividad; gestión de la información; modelos de gestión de la información y, posteriormente, un estudio bibliométrico realizado en los artículos de revistas brasileñas y argentinas en el período de enero de 2007 a enero de 2017. Como resultado, se demuestra que hay una producción considerable sobre la temática, y que se mantiene con el transcurrir de los años. Se considera que existe un interés de los investigadores en cuanto a la importancia de la gestión de la información para el área disciplinar.
\end{abstract}

\begin{abstract}
Understanding how information management is being handled by the area's journals assists both in the growth of the area itself and in the professionals who work in it. Therefore, the general objective of this communication was to carry out a detailed analysis of the articles of the journals with Qualis Capes A1, A2 and B1 strata of the Communication and Information area, addressed to the Information Management, Brazil and Argentina. With regard to the specific objectives, we tried to identify which are the periodicals that most published articles in the theme; Discover in which year there were more publications; Knowing who are the most productive authors; Listing the most commonly used keywords in the articles; To ascertain the methodological procedures used and the way the recovered texts are discussing the information management theme, as well as their models in these contexts. Regarding the methodology, the article is exploratory with a qualitative-quantitative approach based on a bibliographical review on the topics of information and competitiveness; information management; Information management models and, later, a bibliometric study carried out in the articles of Brazilian and Argentine periodicals from January 2007 to January 2017. As results, they show that there is considerable production on the subject and that it is being. Maintained over the years. it is considered that there is the interest of researchers in the importance of information management for the area.
\end{abstract}

Recibido: 28 de mayo de 2017 | Aceptado: 31 de agosto de 2017 | Publicado: 9 de octubre de 2017

Cita sugerida: Santos Neto, J. A. dos, Santos, J. C. dos y Nascimento, N. M. do (2017). Gestión de la información en las revistas de Brasil y Argentina: un análisis de la última década. Palabra Clave (La Plata),7(1), e037.

https://doi.org/10.24215/18539912e037 


\section{Introducción}

La información y el conocimiento son esenciales para el desarrollo de la sociedad y, consecuentemente, de las organizaciones, reduciendo o almacenando incertidumbres cuando son comprendidos como elementos estratégicos de la organización. Además, evidencian al sujeto organizacional su valor y por eso necesitan ser gestionados.

Gestionar información y contextualizarla para facilitar los flujos y procesos organizacionales, se constituyen en acciones informacionales para que las organizaciones actúen con seguridad en el mercado global (Santos y Valentim, 2015). Es decir, la información se torna materia prima importante en las relaciones humanas, sociales, globales y empresariales.

Ante el volumen de información existente en los ambientes organizacionales, se entiende que para alcanzar la ventaja competitiva deseada es necesario que la información sea valorada y "dominada", siendo gestionada de manera eficaz desde su prospección, selección, filtraje, tratamiento, organización, almacenamiento, diseminación y uso, alimentando y consolidando la toma de decisión.

Cuando se trabaja con gestión de la información (GI) en el contexto organizacional, se analiza el ambiente interno y el mercado donde esta organización actúa, es decir, la eficiencia (atender las necesidades de la organización) y la eficacia (alcanzar objetivos propuestos), lo que refuerza la importancia de la sistematización de las informaciones, ya que ella da el apoyo y soporte en las funciones de planeamiento de la empresa.

Cuanto mejor es el tratamiento, la gestión y la organización de la información (elemento cíclico y reutilizable), más rápido se alcanza la competitividad ansiada.

De esa manera, el objetivo de este trabajo es evidenciar por medio de las revistas de dos países (Brasil y Argentina) con estrato Qualis Capes A1, A2 y B1, en las áreas de comunicación e información, cómo la temática GI viene siendo trabajada en estos dos universos por los investigadores del área. También se pone de relieve cómo este tratamiento de la temática potencia la competitividad en el ambiente organizacional. Los objetivos específicos fueron: identificar cuáles son las revistas que más publicaron artículos en la temática; descubrir en qué año hubo un mayor número de publicaciones; conocer quiénes son los autores más productivos; encasillar las palabras-clave más empleadas en los artículos; comprobar los procedimientos metodológicos utilizados y la manera en que los textos recuperados están discutiendo la temática, así como sus modelos en estos contextos.

Investigaciones en esa misma área de actividad ya fueron realizadas y algunas de ellas son indicadas en este artículo, con el objetivo de fundamentar la presente discusión y confrontar los resultados obtenidos. Kleinubing (2010) presentó un estudio bibliométrico de las publicaciones relacionadas con el área de GI de la base de datos Library and Information Science Abstracts (Lisa), en el período de 1984 a 2008, a partir de 138 artículos. Souza y Duarte (2011) mapearon las dimensiones de la GI en los anales del Grupo de Trabajo 4 de la Asociación Nacional de Investigación y Posgrado en Ciencias de la Información en Brasil (Ancib) en el período de 1994 a 2008, analizando 66 trabajos. Kroeff et al. (2015) realizaron un estudio de citaciones de 195 artículos publicados en 
revistas indexadas por la Base de Datos de Revistas en Ciencia de la Información (BRAPCI), también en la temática de la GI, en el periodo de 2000 a 2011.

Se justifica la realización de este estudio, dado que no fueron localizados artículos de revistas que presenten resultados más recientes, es decir, que contemplen el panorama científico de la GI en la Ciencia de la Información (CI) en la última década.

La metodología se centra en un repaso de bibliografía; es un estudio de carácter exploratorio con abordaje cualitativo y cuantitativo, con la finalidad de proponer diálogos en base a la literatura consultada. Se ansía que las reflexiones brinden una mejor comprensión del objeto y fenómenos investigados. Además se espera incitar a nuevos investigadores a discutir acerca de las temáticas abordadas.

\section{Gestión de la Información: algunas consideraciones}

Es muy común la confusión y/o asociación entre las temáticas GI y la gestión del conocimiento (GC). Para Valentim (2004, p. 1, traducción propia) "algunas corrientes funden los modelos de gestión, o confunden, todavía, un modelo con el otro." Sin embargo, según la autora, lo que contribuye para este equívoco es que "las dos gestiones convergen para el hecho de que pretenden apoyar/subsidiar las actividades desarrolladas en el cotidiano, y la toma de decisión en la organización." (Valentim, 2004, p. 1, traducción propia), y por este motivo a continuación se presentan brevemente las definiciones apuntadas por la literatura y que diferencian estos dos procesos de gestión. La GI comprende,

un conjunto de estrategias que visa identificar las necesidades informacionales, mapear los flujos formales de información en los diferentes ambientes de la organización, así como su colecta, filtraje, análisis, organización, almacenaje y diseminación, objetivando apoyar el desarrollo de las actividades cotidianas y la tomada de decisión en el ambiente corporativo (Valentim, 2004, p.1, traducción propia).

El objetivo de la GI es potencializar los recursos informacionales existentes, ampliando la capacidad del aprendizaje organizacional y apoyando el proceso decisorio por medio de las actividades desarrolladas por la organización.

Por otro lado, la GC es un conjunto de estrategias para "crear, adquirir, compartir y utilizar activos de conocimiento, así como establecer flujos que garanticen la información necesaria en el tiempo y formato adecuados, con la finalidad de auxiliar en la generación de ideas, soluciones de problemas y la toma de decisión." (Valentim, 2004 p. 1).

El proceso de gestión del conocimiento involucra personas con sus habilidades y competencias y los procesos que ellas desarrollan. Tales procesos están directamente ligados a la producción de bienes y servicios. Esa producción, para ser competitiva deberá obedecer a las reglas de la utilización perfecta de los recursos y de las inversiones, es decir, productividad. (Corsatto y Hoffmann, 2013, p.21, traducción propia). 
La GI y la GC actúan en los flujos informacionales de la organización. El flujo se divide en dos, flujo informacional informal o no estructurado, que es donde actúa la GC, y el flujo informacional formal o estructurado, que es donde actúa la GI. Los flujos informacionales no estructurados (informales):

se caracterizan, casi siempre, por su invisibilidad, una vez que se constituyen en el resultado de vivencias y experiencias individuales y grupales de los sujetos organizacionales, son apoyados por el aprendizaje organizacional y por el intercambio/sociabilización del conocimiento entre otras personas. Para este tipo de flujo es necesaria la gestión del conocimiento, cuyo trabajo es realizado por todas las personas que actúan en la organización, una vez que la responsabilidad se refiere al intercambio y sociabilización de vivencias y experiencias individuales y grupales. (Valentim, 2013, p. 306, traducción propia).

Los flujos informacionales estructurados (formales), a su vez,

se caracterizan por su visibilidad, se constituyen en el resultado de las actividades y tareas desarrolladas de manera repetitiva en el ambiente organizacional, son apoyados por normas de procedimientos y especificaciones claras, son registrados en diferentes soportes, circulan en distintos medios y, además de eso, existe la gestión de la información por una o varias personas, cuya responsabilidad se refiere al tratamiento, organización, almacenaje, preservación y diseminación de las informaciones que por él pasaron, de manera que el acceso, apropiación y uso puedan de hecho ser efectivos. (Valentim, 2013, p. 306, traducción propia).

Gestionar la información es decidir qué hacer basándose en ella y acerca de ella (Braga, 2000). La GI permitirá a los gestores el planeamiento de estrategias para agregar más valor a las informaciones, transformándolas en el conocimiento necesario para el éxito de las organizaciones.

Davenport y Prusak (1998) afirman que el ambiente de información tiene en cuenta los valores y las creencias organizacionales, visto que la gestión es una actividad que engloba planeamiento, dirección, organización y control, es decir, establece principios, políticas, planes, funciones y actividades buscando la eficiencia para alcanzar los objetivos organizacionales.

La GI es un proceso de gestión; una vez que la obtención de informaciones no puede ser interrumpida, entonces, debe haber un sistema de adquisición continuada en varias actividades, explotación del ambiente informacional, clasificación de la información en una estructura pertinente, formato y estructuración de las informaciones (Davenport y Prusak, 1998).

En esa perspectiva, poseer información sobre el mercado potencializa la competitividad de una determinada organización. Para Beuren (2007) el trípode que sostiene la producción de información es la decisión, la mensura y la información.

Una vez que la empresa reconoce el papel positivo que la información puede representar, cabe a ella reflexionar sobre cuestiones primordiales relacionadas a la creación de procesos eficaces de gestión de la información (...) La gestión de un sistema de información, como un proceso inter funcional vital en la organización, 
puede contribuir para mejorar la utilidad de ese recurso (...) la gestión de la información es, actualmente, tanto del punto de vista académico como de sus aplicaciones en el mundo de los negocios, un tema de mayor relevancia. Dentro del contexto económico, esa función es considerada una de las responsables por el éxito de las organizaciones, sea en el nivel de supervivencia o en el establecimiento de mayor competitividad. (Beuren, 2007, pp. 59-64, traducción propia).

La realidad actual, juntamente con las tecnologías de la información y comunicación (TIC), exige que las organizaciones estén cambiando y adaptándose de tal manera que ellas puedan ser capaces de producir bienes y servicios de información, atendiendo a la necesidad organizacional. En este sentido, se destaca que la gestión estratégica de la información no es algo que posee linealidad sino que, por lo contrario, consiste en algo dinámico y debe ser capaz de percibir las necesidades y los flujos informacionales. Así, la información es un recurso estratégico que, cuando es gestionada por medio de la GI, subsidia la toma de decisión. Además de eso, su gestión permite a los gestores hacer planteos estratégicos apoyando las decisiones de la organización. Así, conocer y analizar los abordajes adoptados por los autores del área de comunicación e información resulta vital para contribuir al crecimiento del área de la CI y de la actuación del profesional en las organizaciones.

\section{Modelos de GI}

Los modelos de GI pueden ser clasificados como teóricos o aplicados. La palabra modelos se caracteriza como una representación de algo, y ante eso, los modelos de GI presentan las directrices de cómo la información debe ser trabajada en los ambientes organizacionales para alcanzar la competitividad ansiada.

Los modelos teóricos de GI son representaciones esquemáticas, controlables y simplificadas, que proporcionan para las organizaciones una visión macro de la realidad. Por otro lado, los modelos aplicados atienden a determinada realidad/organización en sus necesidades y demandas específicas. Las etapas/fases del proceso de GI son denominadas en la literatura como modelo. Tales modelos fueron identificados en las revistas del área de comunicación e información ( $A 1, A 2$ y B1) y los más citados son los de McGee y Prusak (1994), Davenport y Prusak (1998), Davenport (2002) y Choo (2003). De ese modo, con la intención de evidenciar sintéticamente los modelos más citados, la tabla 1 presenta el siguiente panorama.

\section{Tabla 1. Modelos y etapas del proceso de GI}

\begin{tabular}{|l|l|}
\hline \multicolumn{1}{|c|}{ Modelos } & \multicolumn{1}{c|}{ Etapas } \\
\hline & 1 - Necesidad y requisitos de Información; \\
& 2 - Adquisición y colecta de información; \\
McGree y & - Clasificación, almacenamiento y presentación de la \\
Prusak (1994) & información; \\
& 4- Desarrollo de productos y servicios de información; \\
& 5 - Distribución y diseminación de la información; \\
& 6- Análisis y uso de la Información. \\
\hline
\end{tabular}




\begin{tabular}{|c|c|}
\hline $\begin{array}{l}\text { Davenport y } \\
\text { Prusak (1998) }\end{array}$ & $\begin{array}{l}1 \text { - Determinación de las exigencias de información; } \\
2 \text { - Obtención de la información; } \\
3 \text { - Distribución y utilización de la información; } \\
4 \text { - Creación y disposición de productos y servicios de información. }\end{array}$ \\
\hline $\begin{array}{l}\text { Davenport } \\
(2002)\end{array}$ & $\begin{array}{l}1 \text { - Determinación de exigencias de información; } \\
2 \text { - Obtención de la información; } \\
3 \text { - Distribución de la información; } \\
4 \text { - Utilización de la información. }\end{array}$ \\
\hline Choo (2003) & $\begin{array}{l}1 \text { - Necesidad de información; } \\
2 \text { - Adquisición de información; } \\
3 \text { - Organización y almacenamiento de la información; } \\
4 \text { - Productos y servicios de información; } \\
5 \text { - Distribución de información; } \\
6 \text { - Uso de información; } \\
7 \text { - Comportamiento adaptativo. }\end{array}$ \\
\hline $\begin{array}{l}\text { Valentim } \\
(2008)\end{array}$ & $\begin{array}{l}1 \text { - Identificar necesidades/demandas de información; } \\
2 \text { - Mapear y reconocer flujos formales; } \\
3 \text { - Desarrollar la cultura organizacional positiva en relación al } \\
\text { intercambio/socialización de información; } \\
4 \text { - Proporcionar la comunicación informacional de forma eficiente, } \\
\text { utilizando tecnologías de información y comunicación; } \\
5 \text { - Prospección y monitorizar informaciones; } \\
6 \text { - Recolectar, seleccionar y filtrar informaciones; } \\
7 \text { - Tratar, analizar, organizar, almacenar y agregar valor a las } \\
\text { informaciones, utilizando tecnologías de información y } \\
\text { comunicación; } \\
8 \text { - Desarrollar e implantar sistemas informacionales de diferentes } \\
\text { naturalezas, visando el intercambio y el uso de información; } \\
9 \text { - Elaborar productos y servicios informacionales; } \\
10 \text { - Elaborar e implantar normas para la sistematización de la } \\
\text { información producida interna y externamente; } \\
11 \text { - Retroalimentar el ciclo. }\end{array}$ \\
\hline
\end{tabular}

Fuente: Adaptado de Valentim, Jorge y Ceretta Soria (2014) y Souza y Duarte (2011).

Si se observan las informaciones de la tabla 1 , se destaca la existencia de proximidades y complementariedades que atienden la evolución de las necesidades organizacionales. Ante eso, las acciones y relaciones demostradas en los modelos presentados contribuyen para potencializar el diferencial competitivo en el ambiente organizacional, evidenciando el actuar de manera estructurada y anticipada sobre la información para volver el proceso decisorio acertado y anticipado, al igual que destacando la necesidad de múltiples 
habilidades y competencias de los profesionales para lidiar con la GI.

\section{Procedimientos metodológicos}

Como método se utilizó la investigación bibliográfica de naturaleza exploratoria, con abordaje cualitativo y cuantitativo. Según Gil "La investigación bibliográfica es desarrollada a partir de material ya elaborado, constituido principalmente de libros y artículos científicos" (2008, p. 48). De esa manera, se eligió como fuente de investigación para este trabajo los artículos de revistas que son publicados en fascículos y obtienen la contribución de diversos autores con diferentes asuntos abordados y con un alcance definido.

Se realizó también un estudio bibliométrico en el período de enero de 2007 a enero de 2017, es decir, diez años de prospección, en las revistas científicas brasileñas y argentinas con estrato Qualis da Coordenação de Aperfeiçoamento de Pessoal de Nível Superior (CAPES) A1, A2 y B1, en el área de comunicación e información, en el año de evaluación 2015, más específicamente en aquellas revistas que poseen alcance direccionado a la CI. También formaron parte de este corpus las revistas indexadas en la base de datos Latindex, en el área del conocimiento de ciencias sociales y en la subárea CI y Biblioteconomía (solo los vigentes y online), publicadas en Argentina.

Los estudios bibliométricos "[son] procedimientos tangibles y fiables, que utilizan indicadores de producción, enlace y citación, explicitando, más allá de la productividad, la relevancia e impacto de autores, revistas, instituciones, grupos o países" (Oliveira y Grácio, 2011, p. 18, traducción propia). Estos análisis son utilizados para verificar la producción de informaciones científicas, insertando aspectos tales como: publicaciones, autores, palabras-clave y citaciones.

Se optó en este estudio por la adopción de la Ley del Elitismo para determinar los autores más productivos. De acuerdo con Price, "según esta ley, si k representa el número total de contribuyentes en una disciplina, la raíz cuadrada de k representaría la élite del área estudiado" (1963, p. 41, traducción propia).

A partir de la consulta realizada en la Plataforma Sucupira Capes $\underline{1}$ y Latindex $\underline{2}$, se llegó a un total de 27 revistas, de acuerdo con lo que se indica en la tabla 2.

Tabla 2. Revistas, país y estrato Qualis Capes

\begin{tabular}{|l|l|c|}
\hline No & \multicolumn{1}{|c|}{ Nombre de la revista y país } & $\begin{array}{c}\text { Estrato Qualis } \\
\text { Capes }\end{array}$ \\
\cline { 1 - 2 } $\mathbf{1}$ & Informação \& Sociedade - Brasil & \multirow{2}{*}{$\mathrm{A} 1$} \\
\hline $\mathbf{2}$ & Perspectivas em Ciência da Informação - Brasil & \multirow{2}{*}{$\mathrm{A} 2$} \\
\hline $\mathbf{3}$ & Transinformação - Brasil & \\
\cline { 1 - 2 } & $\begin{array}{l}\text { Anais do Museu Paulista: História, Cultura e Material - } \\
\text { Brasil }\end{array}$ & \\
\hline $\mathbf{5}$ & Em Questão - Brasil & \\
\hline $\mathbf{6}$ & Encontros Bibli - Brasil & \\
\hline $\mathbf{7}$ & Informação \& Informação - Brasil & \\
\hline $\mathbf{8}$ & Palabra Clave - Argentina &
\end{tabular}




\begin{tabular}{|l|l|}
\hline $\mathbf{9}$ & Agora - Brasil \\
\hline $\mathbf{1 0}$ & Anais do Museu Histórico Nacional - Brasil \\
\hline $\mathbf{1 1}$ & Arquivo e Administração - Brasil \\
\hline $\mathbf{1 2}$ & Brazilian Journal of Information Science - Brasil \\
\hline $\mathbf{1 3}$ & Ciência da Informação - Brasil \\
\hline $\mathbf{1 4}$ & $\begin{array}{l}\text { INCID: Revista de Documentação e Ciência da Informação } \\
\text { - Brasil }\end{array}$ \\
\hline $\mathbf{1 5}$ & Liinc em Revista - Brasil \\
\hline $\mathbf{1 6}$ & Perspectivas em Gestão \& Conhecimento - Brasil \\
\hline $\mathbf{1 7}$ & $\begin{array}{l}\text { Pesquisa Brasileira em Ciência da Informação e } \\
\text { Biblioteconomia - Brasil }\end{array}$ \\
\hline $\mathbf{1 8}$ & PontodeAcesso - Brasil \\
\hline $\mathbf{1 9}$ & $\begin{array}{l}\text { RBBD. Revista Brasileira de Biblioteconomia e } \\
\text { Documentação - Brasil }\end{array}$ \\
\hline $\mathbf{2 0}$ & $\begin{array}{l}\text { RECIIS. Revista Eletrônica de Comunicação, Informação \& } \\
\text { Inovação em Saúde - Brasil }\end{array}$ \\
\hline $\mathbf{2 1}$ & $\begin{array}{l}\text { Revista Digital de Biblioteconomia e Ciência da Informação } \\
\text { - Brasil }\end{array}$ \\
\hline $\mathbf{2 2}$ & Revista Ibero-Americana de Ciência da Informação - Brasil \\
\hline $\mathbf{2 3}$ & $\begin{array}{l}\text { Tendências da Pesquisa Brasileira em Ciência da } \\
\text { Informação - Brasil }\end{array}$ \\
\hline $\mathbf{2 4}$ & Anuario: Escuela de Archivología - Argentina \\
\hline $\mathbf{2 5}$ & Espectros: Revista Cultural - Argentina \\
\hline $\mathbf{2 6}$ & Información, cultura y sociedad - Argentina \\
\hline $\mathbf{2 7}$ & $\begin{array}{l}\text { Jornadas de Intercambio y Reflexión Acerca de la } \\
\text { Investigación en Bibliotecología - Argentina }\end{array}$ \\
\hline
\end{tabular}

Leyenda: *Revistas recuperadas por la base Latindex y no evaluadas por la Capes.

Fuente: Autoría propia con base en los datos del Portal de la Capes y Latindex, 2017.

De las 27 revistas identificadas, tres de ellas son $A 1$, cinco son $A 2,15$ son $B 1$ y cuatro todavía no fueron evaluadas por la Capes. Se realizaron las búsquedas por el término gestión de la información seleccionando solamente las que presentaron el término en el título de la publicación. Los resultados de este relevamiento son presentados en la próxima sección.

\section{Resultados y discusión}

A partir de las búsquedas realizadas fueron recuperados 80 artículos. Vale resaltar que además de estos, otros documentos también fueron recuperados por los portales de las revistas, como editoriales, resúmenes de tesinas y/o disertaciones, reseñas, etc. No obstante, para este trabajo se consideraron solamente los artículos de revistas.

Según lo ya mencionado, los artículos que compusieron el análisis fueron aquellos que presentaron, obligatoriamente, el término gestión de la información en el título de la publicación. En los 10 años de prospección, es decir, de enero de 2007 a enero de 2017, fueron recuperados 80 artículos en 16 revistas. Por consiguiente, el gráfico que sigue 
presenta la distribución de esos artículos en cada una.

\section{Gráfico 1. Número de artículos recuperados en cada revista}

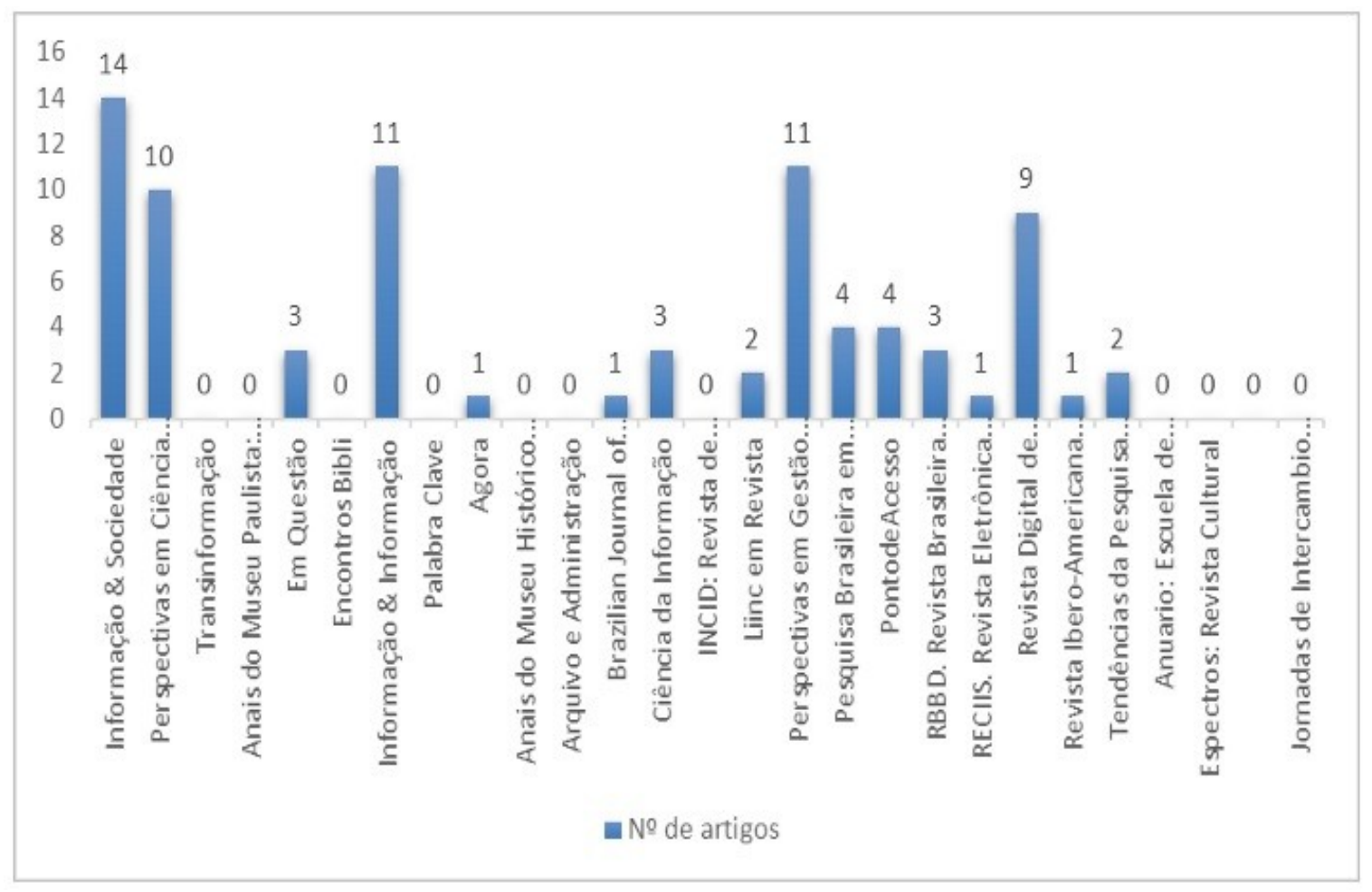

Fuente: elaboración propia.

De acuerdo a lo observado en el gráfico 1 , las revistas que publicaron más artículos sobre GI fueron: Informação \& Sociedade (14); Informação \& Informação (11); Perspectivas em Gestão do Conhecimento (11); Perspectivas em Ciência da Informação (10); y Revista Digital de Biblioteconomia e Ciência da Informação (9). Las demás presentaron entre uno y cuatro artículos. Se resalta que en el relevamiento realizado por Kroeff et al. (2015) en el período de 2000 a 2011, la revista que más publicó artículos fue Ciência da Informação, con 27 trabajos. Al comparar los resultados de la referida investigación con los que fueron presentados en esta comunicación, se verifica que hubo una queda en el número de artículos sobre GI en la revista Ciência da Informação, puesto que en la última década fueron recuperados solamente tres artículos.

Se pone en evidencia, a partir de lo expuesto, que la temática abordada es discutida en el área, aunque Brasil fue quien publicó todos los artículos en diez años, es decir, 80 artículos. En el caso de Argentina, no hay publicaciones identificadas en las revistas del área; no obstante, es necesario tener en consideración que el número de revistas en el país es menor cuando es comparado con Brasil. Otra justificación para el número nulo de artículos recuperados en revistas argentinas es que, quizás, a partir de la delimitación y las estrategias utilizadas en esta comunicación, los artículos que discuten la temática GI no fueron recuperados. Por lo tanto, no se puede afirmar que no existan artículos argentinos que traten sobre el asunto investigado.

En relación al período en que hubo un mayor número de publicaciones, se comprobó un 
equilibrio en el período investigado con una media de nueve artículos por año, siendo 2011 el año en que más artículos fueron publicados (12) y 2012 en el que menos (tres), destacando que hasta el mes de enero de 2017 fueron publicados dos artículos. Kroeff et al. (2015) descubrieron que, hasta el año de 2011, los años en que más hubo publicaciones fueron 2007 y 2008.

Los 80 artículos recuperados fueron publicados por 198 autores de instituciones distintas, siendo que la élite más representativa del universo analizado está compuesta por nueve investigadores vinculados a las siguientes instituciones: Universidad Estadual Paulista (UNESP), Universidad Estadual de Londrina (UEL), Universidad Federal de Minas Gerais (UFMG), Universidad Federal de Santa Catarina (UFSC). Los autores más productivos, que representan la élite científica en el ámbito de los artículos de revistas direccionados a la GI en la última década, élite que puede ser determinada a partir del discurso de Price (1963), son presentados en la tabla 3.

Tabla 3. Autores más productivos

\begin{tabular}{|l|c|c|}
\hline \multicolumn{1}{|c|}{ Autor } & IES & $\begin{array}{c}\text { No de } \\
\text { artículos }\end{array}$ \\
\hline Valentim, Marta Lígia Pomim & UNESP & 5 \\
\hline Barbosa, Ricardo Rodrigues & UFMG & 4 \\
\hline Duarte, Emeide Nóbrega & UFPB & 3 \\
\hline Pinho Neto, Júlio Afonso Sá de & UFPB & 3 \\
\hline Freire, Isa Maria & UFPB & 2 \\
\hline Jorge, Carlos Francisco Bitencourt & UNESP & 2 \\
\hline Porto, Renata Maria Abrantes Baracho & UFMG & 2 \\
\hline Sobral, Natanael Vitor & UFPE & 2 \\
\hline Tomaél, Maria Inês & UEL & 2 \\
\hline Vianna, William Barbosa & UFSC & 2 \\
\hline
\end{tabular}

Fuente: elaboración propia.

Al aplicarse la Ley del Elitismo (Price, 1963) a un universo de k=198 autores, se tendrían que seleccionar 14 como aquellos más productivos. Sin embargo, debido a los índices de producción identificados, fueron seleccionados solamente los 10 autores que presentan como mínimo dos artículos publicados. Si se considera la institución de los autores que más publicaron en esta temática, en estas revistas, se constata que la UNESP es la que posee más participación en esta área, con siete trabajos, seguida por la UFMG con seis, luego la Universidad Federal da Paraiba (UFPB) con cinco y, con dos trabajos cada una, aparecen juntas las instituciones UEL, Universidad Federal de Pernambuco (UFPE) y UFSC.

En la investigación realizada por Santana et al. (2014), fue comprobado que el autor central en el ámbito de GI es Freire, Isa Maria. Kroeff et al. (2015) llegaron a la conclusión de que los autores más productivos fueron Valentim, Marta Lígia Pomim; Barbosa, Ricardo Rodrigues; Freire, Isa Maria y Tomaél, Maria Inês. De este modo, al comparar tales resultados con los aquí presentados, es posible afirmar que el núcleo común de autores más productivos en el ámbito de la GI está compuesto por: Valentim, Marta Lígia Pomim; Duarte, Emeide Nóbrega; Barbosa, Ricardo Rodrigues; Tomaél, Maria Inês, y Freire, Isa Maria. 
En cuanto a las palabras-clave, en los 80 artículos analizados fueron empleados 257 términos diferentes, siendo los términos más citados gestión de la información que obtuvo 63 ocurrencias, gestión del conocimiento con 23 ocurrencias y ciencia de la información con 10 ocurrencias. Kroeff et al. (2015), al analizar las 833 palabras-clave empleadas en los artículos de 2000 a 2011, evidenciaron que el término gestión de la información también fue el más adoptado, con 101 ocurrencias; los demás términos fueron gestión del conocimiento con 37 usos, inteligencia comparativa con 14 y ciencia de la información con 12 ocurrencias. Kleinubing (2010), también verificó que el término gestión de la información obtuvo alto índice de empleo en los artículos, siendo que 117 artículos de los 138 presentados poseen el referido término como palabra clave. Los resultados obtenidos en este estudio van al encuentro de aquellos apuntados por Kleinubing (2010) y Kroeff et al. (2015), puesto que en los análisis realizados las palabras-clave más empleadas por los autores de los artículos son gestión de la información, gestión del conocimiento y ciencia de la información.

En relación al abordaje/método de los artículos, 30 de ellos se presentan exclusivamente teóricos, como las revisiones de literatura e investigaciones bibliográficas. Otros 50 son resultados de investigaciones prácticas, donde ocurrió la aplicación de investigación de hecho (investigación de campo, estudios de caso, etc.); esto evidencia que hay más publicaciones que relatan sobre la aplicación de la GI que trabajos teóricos; no obstante, es importante resaltar que los dos tipos de aportes son importantes para el área.

Los métodos más empleados en los trabajos fueron: estudio de caso único (12), investigación documental (9), estudio de caso múltiple (5), estudio y/o investigación de campo (4). En lo que refiere a las técnicas de recolección más empleadas en los artículos analizados fueron: entrevistas (14); cuestionarios (9); observaciones (5); estudios bibliométricos (3). Esto demuestra que el método más utilizado para reflexionar sobre esta temática en los trabajos aplicados fue el estudio de caso y la técnica de colecta de datos fue la entrevista. La técnica de análisis que demostró ser más utilizada por los autores fue el análisis de contenido.

Finalmente, cuatro artículos poseían en el título o en las palabras-clave el término modelo de gestión de la información; sin embargo, al realizar el análisis de los modelos de gestión adoptados, se observó que en tres de los artículos los modelos teóricos/conceptuales fueron solo mencionados y solamente uno de los artículos adoptaba de hecho el uso de un modelo de GI aplicado.

\section{Consideraciones finales}

Con la prospección y el análisis de los datos se considera que cuestiones importantes merecen ser compartidas. El área de GI en los 10 últimos años está siendo discutida y analizada en el ámbito de la comunidad científica y posee una media de nueve publicaciones por año. Esto último evidencia la importancia de la temática para el área, así como para los profesionales y los ambientes organizacionales, ya que en muchos trabajos existe el relato de la aplicación de los modelos de GI en estos entornos.

En 10 años de producción investigada en el área CI para la GI, se advirtió que Brasil es predominante en el escenario científico en cuanto a las publicaciones en las revistas del 
área, lo que significa que el tema es discutido y profundizado por la comunidad científica. En los estudios de Souza y Duarte (2011) los autores ya resaltaron que la temática GI es tendencia de investigación en la CI.

Por su lado, Argentina no posee ninguna publicación acerca del tema (dentro de los parámetros seleccionados para la elaboración del presente artículo). Una de las justificaciones para esto es que Brasil se constituye como un país geográficamente más grande y también con un índice mayor de revistas del área; es decir, evidentemente la probabilidad de tener un número superior de publicaciones es mayor, no obstante no se esperaba que el resultado de artículos publicados fuera nulo en el ámbito de las revistas existentes en Argentina.

Ante eso, este estudio contribuye para evidenciar la necesidad de publicaciones sobre la temática en Argentina, teniendo en vista que este tema es importante y necesario tanto para las organizaciones como para los profesionales que actúan en distintos ambientes informacionales.

Se concluye que los objetivos fueron alcanzados, siendo la revista Informação \& Sociedade, Qualis A1, aquella que presentó el mayor número de artículos en la temática (14). El año 2011 fue considerado como aquél que tuvo más publicaciones (12). Los autores Valentim, Marta Lígia Pomim (UNESP) y Barbosa, Ricardo Rodrigues (UFMG) se presentaron como los más productivos, con cinco y cuatro artículos respectivamente. Las palabras-clave más adoptadas fueron gestión de la información, gestión del conocimiento y ciencia de la información. El método de investigación más utilizado por los autores fueron el estudio de caso y la entrevista como técnica de colecta de datos.

Es necesario aclarar que algunos textos no presentan un resumen estructurado, es decir, conteniendo las principales secciones del artículo científico: introducción, objetivos, metodología, resultados y conclusiones. Debido a esto, en muchos casos la identificación de tales elementos demandó un análisis minucioso en el cuerpo de los artículos.

En cuanto a los artículos que mencionaron en el título modelos de GI, se observó que la utilización del modelo de hecho no ocurrió; o sea, muchos textos los citaban, pero el uso propiamente dicho de estos modelos ocurre en la minoría de los trabajos.

De este modo, se cree que la relevancia de los trabajos de GI para el área existe, independientemente del tipo de trabajo, sea teórico o práctico, pues ambos presentan sus contribuciones específicas. Todavía es necesario conocer cuáles son las publicaciones que están siendo desarrolladas, incluso para saber qué y por qué se está produciendo, no perdiendo de vista que las publicaciones son un reflejo de las mayores necesidades del área.

\section{Notas}

1 Recuperado de:

https://sucupira.capes.gov.br/sucupira/public/consultas/coleta/veiculoPublicacaoQualis/list aConsultaGeralPeriodicos.jsf.

$\underline{2}$ Recuperado de: http://www.latindex.org/latindex/inicio. 


\section{Referencias bibliográficas}

Braga, A. (2000). A gestão da informação. Millenium Internet, Viseu, 19. Recuperado de http://www.ipv.pt/millenium/19 arq1.htm

Beuren, I. M. (2007). Gerenciamento da informação: um recurso estratégico no processo de gestão empresarial. São Paulo: Atlas.

Corsatto, C. A. y Hoffmann, W. A. M. (2013). Gestão do conhecimento e inteligência competitiva: delineamento de estratégias de competitividade e inovação para pequenas empresas. Encontros bibli: revista eletrônica de biblioteconomia e ciência da informação, 18(38), 19-36. Recuperado de https://periodicos.ufsc.br/index.php/eb/article/view/15182924.2013v18n38p19/25955

Choo, C. W. (2003). A organização do conhecimento: como as organizações usam a informação para criar significado, construir conhecimento e tomar decisões. São Paulo: SENAC.

Davenport, T. H. (2002). Ecologia da Informação: por que só a tecnologia não basta para o sucesso na era da informação. São Paulo: Futura.

Davenport, T. H. y Prusak, L. (1998). Ecologia da informação: por que só a tecnologia não basta para o sucesso na era da informação. São Paulo: Futura.

Gil, A. C. (2008). Métodos e técnicas de pesquisa social. São Paulo: Atlas.

Kleinubing, L. da S. (2010). Análise bibliométrica da produção científica em gestão da informação na base de dados lisa. RDBCI: Revista digital de biblioteconomia e ciência da informação, 8(1), 1-11. Recuperado de https://periodicos.sbu.unicamp.br/ojs/index.php/rdbci/article/view/1943

Kroeff, M. S. et al. (2015). Análise de citações dos artigos publicados em revistas da área da Ciência da Informação que versam sobre gestão da informação. RBBD: revista brasileira de biblioteconomia e documentação, 11(1), 41-65. Recuperado de https://rbbd.febab.org.br/rbbd/article/view/301/397

McGee, J. y Prusak, L. (1994) Gerenciamento estratégico da informação: aumente a competitividade e a eficiência de sua empresa utilizando a informação como uma ferramenta estratégica. Rio de Janeiro: Campus.

Oliveira, E. F. T. de y Grácio, M. C. C. (2011). Indicadores bibliométricos em ciência da informação: análise dos pesquisadores mais produtivos no tema estudos métricos na base Scopus. Perspectiva em Ciência da Informação, 16(4), 16-28. Recuperado de http://portaldeperiodicos.eci.ufmg.br/index.php/pci/article/view/1299/969

Price, J. D. de S. (1963). Litle science, big science. New York: Columbia University Press.

Santana, G. A. et al. (2014). Indicadores dos grupos de pesquisa da área de Gestão da Informação na Região Nordeste: um enfoque para a colaboração em artigos de periódicos. Em Questão: revista da Faculdade de Biblioteconomia e Comunicação da UFRGS, 20(3), 229-252. Recuperado de http://seer.ufrgs.br/index.php/EmQuestao/article/view/49322/32437 
Santos, J. C. y Valentim, M. L. (2015). Gestão da informação em ambientes organizacionais: em foco o setor têxtil e vestuário. Informação e profissões, 4(1), 56-81. Recuperado

http://www.uel.br/revistas/uel/index.php/infoprof/article/view/23542/17205

Souza, I. G. C. de O. y Duarte, E. N. (2011). Dimensões de um modelo de gestão da informação no campo da Ciência da Informação: uma revelação da produção científica do Enancib. Liinc em revista, 7(1), 152-169. Recuperado de http://www.repositorio.ufc.br/bitstream/riufc/4062/1/2011 art IGCOsouza.pdf

Valentim, M. L. P. (2004). Em busca da vantagem competitiva. InfoHome, Londrina. Recuperado de http://www.ofaj.com.br/colunas conteudo.php?cod=87

Valentim, M. L. P. (2013). Ambientes e fluxos de informação em contextos empresariais: o caso do setor cárnico de Salamanca/Espanha. Brazilian journal of information science, 7(especial), 299-323. Recuperado de http://www2.marilia.unesp.br/revistas/index.php/bjis/article/view/3130/2475

Valentim, M. L. P. y Jorge, C. F. B. y Ceretta Soria, M. G. (2014). Contribuição da competência em informação para os processos de gestão da informação e do conhecimento. Em questão: revista da Faculdade de Biblioteconomia e Comunicação da UFRGS, 20(2), 208-230. Recuperado de http://seer.ufrgs.br/index.php/EmQuestao/article/view/48642/32122 